\title{
Conhecimento sobre saúde bucal e práticas desenvolvidas por professores do ensino fundamental e médio
}

\author{
Oral health knowledge and practices developed by teachers of elementary \\ and secondary local schools
}

\author{
Cléa Adas Saliba Garbin* \\ Tânia Adas Saliba Rovida** \\ Luis Fernando Dahmer Peruchini*** \\ Ronald Jefferson Martins
}

\section{Resumo}

Introdução: a educação em saúde bucal é um processo que visa a transformar atitudes e comportamentos e formar hábitos em benefício da saúde do indivíduo. Para que isso ocorra, fazem-se necessários um reforço e uma repetição contínua, possíveis no ambiente escolar. Objetivo: analisar o conhecimento dos professores sobre saúde bucal, as práticas desenvolvidas no ambiente escolar, bem como a participação do cirurgião-dentista na orientação dos professores de ensino fundamental e médio. Sujeitos e método: utilizou-se um questionário semiestruturado, com perguntas abertas e fechadas sobre a formação do professor, orientação recebida sobre saúde bucal, desenvolvimento de temas sobre saúde bucal em sala de aula e conhecimento acerca do assunto. Resultados: do total de entrevistados $(n=221)$, apenas $77(34,8 \%)$ afirmaram que tiveram orientação sobre saúde bucal na sua formação para professor. Setenta $(31,7 \%)$ disseram não desenvolver temas sobre saúde bucal em sala de aula e 91 (41,2\%) que não existia interação entre dentista e professor. Observou-se deficiência no conhecimento dos professores sobre determinados assuntos relacionados à saúde bucal. Houve associação estatística significativa entre orientação do dentista aos professores e o desenvolvimento de ações de saúde bucal, bem como o conhecimento do professor sobre o local de erupção do primeiro molar permanente $(p<0,01)$. Conclusão: apesar de a maior parte dos educadores desenvolver práticas educativas sobre saúde bucal no ambiente escolar, existe deficiência no entendimento de determinados assuntos pelos professores, fazendo-se necessário maior participação do cirurgião-dentista na transmissão de conhecimentos técnicos e científicos aos educadores.

Palavras-chave: Docentes. Conhecimento. Educação em saúde. Saúde bucal.

\section{Introdução}

A legislação brasileira sugere que as ações de promoção da saúde devam ser realizadas por meio da educação, do engajamento em novos estilos de vida e do desenvolvimento intelectual do indivíduo, permitindo a conclusão que o melhor lugar para aprender e reforçar conceitos e bons hábitos de saúde é nas escolas ${ }^{1}$. Existe o desafio de tornar as práticas educativas e preventivas em saúde um cotidiano didático-pedagógico dentro das instituições de ensino, por meio da cooperação entre os setores da educação e da saúde ${ }^{2,3}$.

Professora adjunta do Programa de Pós-graduação em Odontologia Preventiva e Social da Faculdade de Odontologia de Araçatuba, Universidade Estadual Paulista,

Professores Assistentes Doutores do Programa de Pós-graduação em Odontologia Preventiva e Social da Faculdade de Odontologia de Araçatuba, Universidade Estadual Paulista, Brasil.

** Mestrando do Programa de Pós-Graduação em Odontologia Preventiva e Social da Faculdade de Odontologia de Araçatuba, Universidade Estadual Paulista, Brasil. 
Em especial, a educação em saúde bucal é importante por permitir ao indivíduo o acesso ao conhecimento sobre as doenças bucais que podem acometê-lo e seu curso natural, bem como sobre a melhor forma para sua prevenção. Nessa perspectiva, o professor torna-se uma importante ferramenta, por estar desenvolvendo junto ao aluno um trabalho contínuo e sistematizado na infância, que é o período da vida em que a criança se encontra mais apta a agregar conhecimentos, agindo, dessa forma, como modelador de comportamentos na saúde do indivíduo ${ }^{1,4}$. Na educação em saúde bucal, ao professor cabe ser o elo entre o cirurgião-dentista e o aluno, devido ao vínculo maior que possui com os escolares ${ }^{5}$. Por sua vez, o papel de capacitar os educadores é do cirurgião-dentista, que possui o conhecimento técnico e científico sobre o assunto.

A família, baseada na figura dos pais ou do responsável pela criança, é outro forte ponto de apoio na construção dos bons hábitos de higiene, sendo necessário que estes estejam aptos e motivados para essa função. Entretanto, muitos não possuem essa instrução, fazendo do professor um instrumento ainda mais importante na construção do conhecimento, no repasse de informações aos pais ou responsáveis, ou, então, motivando-os a trabalhar em casa práticas de saúde bucal, figurando como o "dentista de todos os dias" no núcleo familiar 6 .

Com base no que foi exposto, objetivou-se neste trabalho analisar o conhecimento dos professores sobre saúde bucal, as práticas desenvolvidas no ambiente escolar, bem como a participação do cirurgião-dentista na orientação dos professores de ensino fundamental e médio de três municípios da região oeste de Santa Catarina, Brasil.

\section{Sujeitos e método}

O presente estudo foi aprovado pelo Comitê de Ética em Pesquisa em Seres Humanos da Faculdade de Odontologia de Araçatuba, processo FOA - 01461/2011. Trata-se de um estudo analítico de base populacional, cujo instrumento de pesquisa foi um questionário de autopreenchimento composto de perguntas abertas e fechadas.

A pesquisa foi desenvolvida com todos os professores das instituições públicas e particulares dos municípios que fazem parte do perímetro urbano de Joaçaba, Santa Catarina, Brasil, a saber, Joaçaba, Herval d'Oeste e Luzerna. Os três municípios estão situados no meio oeste de Santa Catarina, na região pertencente à $9^{\text {a }}$ Gerência Regional de Educação (GERED) do estado. Apresentam juntos uma população de aproximadamente 50 mil habitantes, sendo considerados como municípios de pequeno porte. Possuem 22 escolas públicas no total, sendo dez municipais e oito estaduais, além de quatro instituições particulares de ensino fundamental a médio.
Inicialmente, as escolas foram visitadas pelo pesquisador, que explicou os objetivos e a metodologia do estudo para os diretores, além do posterior uso dos dados coletados, com o propósito de obter suas autorizações para a realização da pesquisa. Previamente à execução do trabalho, foi realizado um estudo piloto com 30 professores da rede pública em outro município que não está entre os integrantes da pesquisa. Os resultados permitiram adequar os questionários à realidade local, trabalhando com as respostas mais prevalentes nas questões fechadas e optando-se por questões abertas onde um número muito variado de respostas foi observado.

Utilizou-se um questionário semiestruturado, com perguntas abertas e fechadas sobre a formação do professor, realização de pós-graduação, orientação recebida sobre saúde bucal durante sua formação, desenvolvimento de temas de saúde bucal em sala de aula, ações e atividades conjuntas entre dentistas e professores e conhecimentos específicos do assunto.

A direção das instituições ficou responsável pela entrega dos questionários, dos Termos de Consentimento Livre e Esclarecido (TCLEs), além de carta que explicava a importância da pesquisa e o procedimento para a resposta dos questionários. Participaram do trabalho somente os professores que assinaram o TCLE.

Cada instituição foi visitada por três vezes para o recolhimento do material, sendo a primeira após um mês a partir da entrega, a segunda após um mês e meio e a terceira após dois meses. Os dados coletados foram tabulados no programa Epi Info, versão 3.5.3 e apresentados em frequências absolutas e percentuais. A análise estatística foi realizada por meio de testes não paramétricos (qui-quadrado e exato de Fisher) com nível de significância de 5\%.

\section{Resultados}

Fizeram parte da pesquisa $21(95,5 \%)$ escolas, tendo apenas uma instituição de ensino particular recusado-se a participar. Do total de 392 professores, $221(56,4 \%)$ responderam aos questionários e assinaram o TCLE. Esses professores atuavam desde o ensino fundamental até o ensino médio. Vinte e cinco $(11,3 \%)$ lecionavam no ensino fundamental inteiro, $116(52,5 \%)$ nas séries iniciais ( $1^{\circ}$ ao $5^{\circ}$ ano), $40(18,1 \%)$ nas finais $\left(6^{\circ}\right.$ ao $9^{\circ}$ ano), $23(10,4 \%)$ trabalhavam com alunos do ensino fundamental e médio e $17(7,7 \%)$ apenas com o ensino médio.

Quanto ao desenvolvimento de ações e atividades de promoção de saúde bucal na escola, 149 (68\%) professores responderam existir tais práticas na instituição em que lecionavam e 70 (32\%) afirmaram não existir (Tab. 1). 
Tabela 1 - Distribuição numérica e percentual dos professores segundo o desenvolvimento de ações de promoção de saúde bucal na escola e a orientação do cirurgião-dentista $(n=219)$

\begin{tabular}{|c|c|c|c|c|c|c|}
\hline \multirow{3}{*}{$\begin{array}{c}\text { Desenvolvimento de ações de } \\
\text { saúde bucal }\end{array}$} & \multicolumn{4}{|c|}{ Orientação do cirurgião-dentista } & \multirow{2}{*}{\multicolumn{2}{|c|}{ Total }} \\
\hline & \multicolumn{2}{|c|}{ Sim } & \multicolumn{2}{|c|}{ Não } & & \\
\hline & $\mathrm{n}$ & $\%$ & $\mathrm{n}$ & $\%$ & $\mathrm{n}$ & $\%$ \\
\hline Sim & 36 & 24,2 & 113 & 75,8 & 149 & 100,0 \\
\hline Não & 5 & 7,1 & 65 & 92,9 & 70 & 100,0 \\
\hline
\end{tabular}

$\mathrm{p}<0,01$

No que diz respeito ao desenvolvimento do assunto saúde bucal em sala de aula, a maioria dos professores - $149(67,4 \%)$ - afirmou que trabalhava o tema com os alunos, $70(31,7 \%)$ disseram não fazê-lo e $2(0,9 \%)$ não responderam. As disciplinas de ciências e de biologia são as principais responsáveis por promover saúde e reforçar os bons hábitos de higiene, pois faz parte do seu conteúdo curricular trabalhar o corpo humano, as doenças que podem atingi-lo e formas de prevenção. Neste estudo, participaram $131(59,3 \%)$ professores de ciências ou biologia, dentre os quais apenas $21(16,0 \%)$ tinham graduação em Ciências Biológicas (Tab. 2).

Tabela 2 - Distribuição numérica e percentual dos professores segundo o desenvolvimento de temas sobre saúde bucal em sala de aula e a disciplina responsável $(n=219)$

\begin{tabular}{|c|c|c|c|c|c|c|}
\hline \multirow{3}{*}{ Professor de ciências ou biologia } & \multicolumn{4}{|c|}{ Desenvolvimento de temas sobre saúde bucal na sala de aula } & \multirow{2}{*}{\multicolumn{2}{|c|}{ Total }} \\
\hline & \multicolumn{2}{|c|}{ Sim } & \multicolumn{2}{|c|}{ Não } & & \\
\hline & $\mathrm{n}$ & $\%$ & $\mathrm{n}$ & $\%$ & $\mathrm{n}$ & $\%$ \\
\hline Sim & 116 & 88,5 & 15 & 11,5 & 131 & 100,0 \\
\hline Não & 33 & 37,5 & 55 & 62,5 & 88 & 100,0 \\
\hline
\end{tabular}

$\mathrm{p}<0,01$

Os professores foram questionados sobre temas relacionados à saúde bucal. Da amostra de 221 sujeitos, $174(78,1 \%)$ não descreveram corretamente a função do flúor na prevenção da cárie dentária; $172(77,8 \%)$ afirmaram não saber ou que teriam dificuldades para administrar um caso de avulsão dentária; $163(73,8 \%)$ não descreveram a placa dental corretamente; $158(71,5 \%)$ não sabiam dizer que cuidados tomar em relação ao pedaço de dente fraturado no caso da ocorrência de fratura dental; 154 $(69,7 \%)$ não sabiam que o primeiro molar permanente erupciona atrás do segundo molar decíduo; $148(67,0 \%)$ erraram ao afirmar que o primeiro sinal da gengiva doente é a sua coloração avermelhada; $112(50,7 \%)$ descreveram a cárie como não sendo transmissível, ou, ainda, como não sendo uma doença; 101 (45,7\%) afirmaram não fazer diferença a concentração de flúor para que se tenha a preven- ção da cárie dentária; $84(38,0 \%)$ acreditavam que a cárie dentária era uma patologia que independia da presença do microrganismo; $72(32,6 \%)$ consideravam a escova de cerdas duras e médias como a mais indicada para a escovação dental; $56(25,4 \%)$ não sabiam qual a quantidade de pasta recomendada; $56(11,3 \%)$ acreditavam que a quantidade de açucar é mais prejudicial que a frequência de ingestão; e 21 $(9,5 \%)$ entendiam que em outros períodos que não o noturno a escovação era mais importante.

Os resultados evidenciaram que dos $149(67,4 \%)$ profissionais que afirmaram desenvolver ações de saúde bucal em sala de aula, apenas $36(24,2 \%)$ receberam algum tipo de treinamento por parte de cirurgiões-dentistas. A relação entre a orientação do cirurgião-dentista aos professores e o conhecimento sobre determinados assuntos de saúde bucal pode ser observada na Tabela 3. 
Tabela 3 - Distribuição numérica e percentual do conhecimento sobre saúde bucal dos professores segundo a presença ou ausência de orientação do cirurgião-dentista

\begin{tabular}{|c|c|c|c|c|c|c|c|}
\hline \multirow{2}{*}{\multicolumn{2}{|c|}{ Pergunta / Resposta }} & \multicolumn{4}{|c|}{ Orientação do cirurgião-dentista } & \multirow{2}{*}{\multicolumn{2}{|c|}{ Total }} \\
\hline & & \multicolumn{2}{|c|}{ Sim } & \multicolumn{2}{|c|}{ Não } & & \\
\hline & & $\mathrm{n}$ & $\%$ & $\mathrm{n}$ & $\%$ & $\frac{n}{109}$ & $\begin{array}{c}\% \\
100,0\end{array}$ \\
\hline O que é cárie dental? & $\begin{array}{l}\text { Certa } \\
\text { Errada } \\
\text { Total }\end{array}$ & $\begin{array}{l}22 \\
14\end{array}$ & $\begin{array}{l}20,2 \\
18,4\end{array}$ & $\begin{array}{l}87 \\
62\end{array}$ & $\begin{array}{l}79,8 \\
81,6\end{array}$ & $\begin{array}{c}109 \\
76 \\
n=185\end{array}$ & $\begin{array}{c}100,0 \\
100,0 \\
p=0,91\end{array}$ \\
\hline $\begin{array}{l}\text { Para que ocorra a cárie } \\
\text { dental o MO deve estar } \\
\text { presente? }\end{array}$ & $\begin{array}{l}\text { Certa } \\
\text { Errada } \\
\text { Total }\end{array}$ & $\begin{array}{l}34 \\
02\end{array}$ & $\begin{array}{c}24,8 \\
8,0\end{array}$ & $\begin{array}{c}103 \\
23\end{array}$ & $\begin{array}{l}75,2 \\
92,0\end{array}$ & $\begin{array}{c}137 \\
25 \\
n=162\end{array}$ & $\begin{array}{c}100,0 \\
100,0 \\
p=0,10\end{array}$ \\
\hline $\begin{array}{l}\text { Você sabe o que é placa } \\
\text { dental? }\end{array}$ & $\begin{array}{l}\text { Certa } \\
\text { Errada } \\
\text { Total }\end{array}$ & $\begin{array}{l}14 \\
28\end{array}$ & $\begin{array}{l}24,1 \\
18,1\end{array}$ & $\begin{array}{c}44 \\
127\end{array}$ & $\begin{array}{l}75,9 \\
81,9\end{array}$ & $\begin{array}{c}58 \\
155 \\
n=213\end{array}$ & $\begin{array}{c}100,0 \\
100,0 \\
p=0,42\end{array}$ \\
\hline $\begin{array}{l}\text { Qual o principal sinal } \\
\text { que uma gengiva doente } \\
\text { apresenta? }\end{array}$ & $\begin{array}{l}\text { Certa } \\
\text { Errada } \\
\text { Total }\end{array}$ & $\begin{array}{l}15 \\
27\end{array}$ & $\begin{array}{l}20,5 \\
18,2\end{array}$ & $\begin{array}{c}58 \\
121\end{array}$ & $\begin{array}{l}79,5 \\
81,8\end{array}$ & $\begin{array}{c}73 \\
148 \\
n=221\end{array}$ & $\begin{array}{c}100,0 \\
100,0 \\
p=0,81\end{array}$ \\
\hline $\begin{array}{l}\text { Você sabe qual a } \\
\text { importância da utilização } \\
\text { do flúor na prevenção da } \\
\text { cárie dentária? }\end{array}$ & $\begin{array}{l}\text { Certa } \\
\text { Errada } \\
\text { Total }\end{array}$ & $\begin{array}{c}8 \\
30\end{array}$ & $\begin{array}{l}17,0 \\
20,8\end{array}$ & $\begin{array}{c}39 \\
114\end{array}$ & $\begin{array}{l}83,0 \\
79,2\end{array}$ & $\begin{array}{c}47 \\
144 \\
n=191\end{array}$ & $\begin{array}{c}100,0 \\
100,0 \\
p=0,72\end{array}$ \\
\hline $\begin{array}{l}\text { Qualquer concentração } \\
\text { de flúor protege os dentes? }\end{array}$ & $\begin{array}{l}\text { Certa } \\
\text { Errada } \\
\text { Total }\end{array}$ & $\begin{array}{l}26 \\
14\end{array}$ & $\begin{array}{l}21,7 \\
14,7\end{array}$ & $\begin{array}{l}94 \\
81\end{array}$ & $\begin{array}{l}78,3 \\
85,3\end{array}$ & $\begin{array}{c}120 \\
95 \\
n=215\end{array}$ & $\begin{array}{c}100,0 \\
100,0 \\
p=0,26\end{array}$ \\
\hline $\begin{array}{l}\text { Qual é a quantidade de } \\
\text { pasta dental que deve ser } \\
\text { colocada na escova de } \\
\text { dentes? }\end{array}$ & $\begin{array}{l}\text { Certa } \\
\text { Errada } \\
\text { Total }\end{array}$ & $\begin{array}{l}29 \\
11\end{array}$ & $\begin{array}{l}17,6 \\
21,2\end{array}$ & $\begin{array}{c}136 \\
41\end{array}$ & $\begin{array}{l}82,4 \\
78,8\end{array}$ & $\begin{array}{c}165 \\
52 \\
n=217\end{array}$ & $\begin{array}{c}100,0 \\
100,0 \\
p=0,70\end{array}$ \\
\hline $\begin{array}{l}\text { Quanto ao açúcar, o que } \\
\text { é mais prejudicial para os } \\
\text { dentes, a quantidade ou a } \\
\text { frequência? }\end{array}$ & $\begin{array}{l}\text { Certa } \\
\text { Errada } \\
\text { Total }\end{array}$ & $\begin{array}{c}38 \\
4\end{array}$ & $\begin{array}{l}19,4 \\
16,0\end{array}$ & $\begin{array}{c}158 \\
21\end{array}$ & $\begin{array}{l}80,6 \\
84,0\end{array}$ & $\begin{array}{c}196 \\
25 \\
n=221\end{array}$ & $\begin{array}{c}100,0 \\
100,0 \\
p=0,89 \\
\text { Fisher }=0,46\end{array}$ \\
\hline $\begin{array}{l}\text { Em qual período deve-se } \\
\text { dar maior atenção para a } \\
\text { escovação dentária? }\end{array}$ & $\begin{array}{l}\text { Certa } \\
\text { Errada } \\
\text { Total }\end{array}$ & $\begin{array}{l}40 \\
02\end{array}$ & $\begin{array}{c}20,0 \\
9,5\end{array}$ & $\begin{array}{c}160 \\
19\end{array}$ & $\begin{array}{l}80,0 \\
90,5\end{array}$ & $\begin{array}{c}200 \\
21 \\
n=221\end{array}$ & $\begin{array}{c}100,0 \\
100,0 \\
p=0,38 \\
\text { Fisher }=0,19\end{array}$ \\
\hline $\begin{array}{l}\text { O primeiro molar, } \\
\text { conhecido como molar } \\
\text { dos } 6 \text { anos, erupciona... } \\
\text { Resposta: }\end{array}$ & $\begin{array}{l}\text { Certa } \\
\text { Errada } \\
\text { Total }\end{array}$ & $\begin{array}{l}18 \\
17\end{array}$ & $\begin{array}{l}26,9 \\
12,1\end{array}$ & $\begin{array}{c}49 \\
123\end{array}$ & $\begin{array}{l}73,1 \\
87,9\end{array}$ & $\begin{array}{c}67 \\
140 \\
n=207\end{array}$ & $\begin{array}{c}100,0 \\
100,0 \\
p=0,01\end{array}$ \\
\hline
\end{tabular}

\section{Discussão}

As pesquisas com questionários representam um desafio por usualmente apresentarem uma baixa taxa de resposta da população estudada. $\mathrm{O}$ valor obtido neste estudo está próximo ao de um trabalho com professores do ensino fundamental ${ }^{7}$ e bem acima do resultado encontrado por outro autor ${ }^{8}$. Acreditamos que a porcentagem de resposta não foi mais expressiva pelo fato de a pesquisa ter sido execu- tada em um período em que os professores da rede estadual de ensino de Santa Catarina entraram em greve, pois a menor taxa de questionários respondidos foi encontrada justamente entre esse grupo.

Os profissionais da educação vêm se capacitando cada vez mais, optando por cursos reconhecidos, como especializações e mestrados. Nos três municípios estudados, a maioria dos profissionais possuía pós-graduação em alguma das áreas do ensino, sendo a mais procurada a especialização em psicope- 
dagogia. Entretanto, observamos que, entre esses educadores, poucos haviam recebido instruções sobre saúde bucal durante a pós-graduação. Outra pesquisa que também avaliou o nível educacional dos professores mostrou resultado diferente quanto ao seu nível de estudo, variando entre $17 \%$ e $81 \%$ de profissionais com especialização9 .

A escola é o local mais indicado para a contrução dos hábitos de higiene bucal. Nas instituições estudadas, verificamos que mais da metade dos professores trabalhavam o tema em sala de aula, número inferior ao encontrado em outro estudo com professores do ensino fundamental, onde se observou que quase a totalidade dos professores trabalhavam o tema saúde bucal em sala de aula? Valores menores também foram encontrados em outros estudos ${ }^{9}$, nos quais apenas cerca de um terço dos educadores afirmou trabalhar temas relacionados à saúde bucal com os alunos e mais da metade relatou que o profissional mais indicado para repassar esse conteúdo aos alunos seria o cirurgião-dentista, e não o professor.

Um dado preocupante observado no estudo foi o alto número de professores que não haviam recebido qualquer forma de instrução sobre saúde bucal na sua formação, e, dos professores que receberam, menos de um quinto afirmou que havia sido de um profissional da área odontológica. Valores baixos da participação do dentista no processo educativo também foram encontrados em outras pesquisas ${ }^{10,11}$. Esses resultados contrastam com os altos níveis de envolvimento do cirurgião-dentista encontrados em outros estudos ${ }^{5,12}$. A presença do profissional da Odontologia é indispensável no processo de transmissão de conhecimentos e incorporação de hábitos em prol da saúde bucal dos escolares e professores. Porém, o estímulo e a continuidade na motivação das crianças cabem ao professor, uma vez que este exerce grande influência sobre os alunos ${ }^{13}$. Para isso, é necessário que os educadores encarregados desse ensino e motivação tenham cuidados com a própria saúde bucal e possuam conhecimento sobre esses temas, para que assim hábitos corretos possam ser criados pelos educandos ${ }^{8}$.

O professor, ao desenvolver com o aluno temas relacionados à saúde bucal em sala de aula, deve utilizar diferentes instrumentos educativos que possibilitarão o aprendizado, a fim de que não se estabeleça a monotonia, pois, caso contrário, levará, principalmente, o aluno adolescente à indiferença com relação ao que está aprendendo ${ }^{14}$. Dessa forma, a saúde bucal deve ser incluída no conteúdo das disciplinas e trabalhada de maneira participativa, através de atividades lúdicas e jogos pedagógicos ${ }^{15}$.

Os aspectos do dia a dia do aluno, de natureza cultural e antropológica, devem ser inseridos no trabalho de educação em saúde. As palestras, teatros e brincadeiras chamam a atenção da criança, que assimila a informação enquanto se diverte. $O$ próximo passo é a fixação e a aplicação desse conteúdo por meio da escovação dental supervisionada e da revelação do biofilme dental. A confirmação do sucesso do trabalho de educação é percebida na redução do nível de placa dental e no repasse das informações pelas crianças a seus familiares em casa ${ }^{14,16,17}$.

No presente trabalho, verificou-se que a maioria dos professores afirmou que a cárie dentária não é uma doença e que também não é transmissível. Resultados preocupantes também foram encontrados em outro trabalho ${ }^{18}$, em que mais da metade dos profissionais disseram que a cárie não era transmissível. Na mesma pesquisa ${ }^{18}$, o registro de que a cárie dentária não era uma doença foi feito por um quarto dos participantes.

Embora os professores tenham um conceito formado sobre placa dental adquirido no decorrer da vida acadêmica e profissional, ao pedir para que eles a descrevessem, tivemos como resposta que se trataria de restos alimentares que ficam aderidos aos dentes quando não é realizada a escovação, corroborando o achado de outros autores ${ }^{9,19}$. Essa visão simplista de placa dental pode ser culpa do próprio dentista, devido à linguagem popular que utiliza, ao definir a placa como "sugeirinha". A placa dental não é formada apenas por restos alimentares, mas sim por uma complexa comunidade de microrganismos indefinidos e associados aderidos à superficíe do esmalte dental ${ }^{20}$.

O conceito errôneo da placa dental pode justificar o achado deste trabalho, no qual parte dos professores não viam ou não sabiam que existia uma relação entre a cárie e a presença de microrganismos. Em outro estudo com professores de escolas municipais de uma localidade em Pernambuco, $69,6 \%$ deles disseram que a causa da cárie seria a escovação incorreta, porém apenas $1,2 \%$ a relacionaram com bactérias ${ }^{9}$.

A quantidade de dentifrício deve ser observada com muita atenção, principalmente na primeira infância, devido ao risco de fluorose. Os achados desta pesquisa evidenciaram que os profissionais sabem da importância do uso controlado de creme dental, e valores positivos também foram encontrados em outro estudo com professores ${ }^{7}$.

A escolha da escova dental é, na maioria das vezes, influenciada pelo poder de marketing e pelo custo, porém o que deve ser levado em consideração no momento da compra são as especificações técnicas e o conforto que esse instrumento proporciona para o indivíduo no momento da escovação. As características ideais são ter as cerdas macias ou extramacias e a cabeça pequena, pois isso torna mais dificil causar injúrias ao periodonto e permite uma melhor limpeza dos dentes superiores e posteriores (molares). A opção pela escova mais macia foi observada no presente estudo e entre a maioria dos professores em outra pesquisa ${ }^{21}$, mostrando que muito do que é passado à população é assimilado e transmitido. 
Como afirmou a maior parte dos professores no presente trabalho, o período no qual se deve prestar mais atenção à escovação é antes de dormir, devido à diminuição da atividade corporal, inclusive do fluxo salivar, que acaba permitindo uma maior ação das bactérias bucais. Esse achado concorda com o de outros estudos ${ }^{19,21}$.

O questionamento sobre a forma de atuação do flúor na prevenção da cárie dentária mostrou falta de um conhecimento consistente por parte dos educadores, pois, embora saibam que o flúor é importante, a maioria não entende como se dá seu mecanismo de ação. Outros estudos em diversas regiões do Brasil corroboram o nosso achado ${ }^{5,7,10,21}$.

Durante muito tempo, o cirurgião-dentista abordou como principal tema de saúde bucal a cárie dentária, deixando outros assuntos em segundo plano. Talvez seja essa a causa do baixo índice de acerto com relação às perguntas referentes a trauma dental. Nesses casos, a realização de ações corretas e o período de tempo gasto até o atendimento no consultório odontológico são fundamentais para o sucesso do tratamento ${ }^{22}$. Observou-se, no presente trabalho, que muitos professores não sabiam qual atitude a ser tomada, tendo tido ou não orientação do cirurgião-dentista. Em outro estudo, foram encontrados valores bem superiores aos nossos acha$\operatorname{dos}^{11}$, provavelmente pela presença, na pesquisa, de professores de turmas avançadas do ensino fundamental e do ensino médio.

O desenvolvimento do tema saúde bucal é uma realidade nas salas de aula dos professores que participaram da pesquisa, porém a alta taxa de respostas incorretas para questões básicas como cárie dentária e uso de fluoretos coloca em risco as iniciativas tomadas. É necessário que os cirurgiões-dentistas se envolvam ativamente no processo de transmissão de conhecimento para essa população, transformando os conhecimentos empíricos, como a ideia de "sugeirinha" como conceito de placa dental, para o conhecimento científico, a fim de auxiliar os professores na transmissão de informações corretas aos alunos e, dessa forma, subsidiá-los na formação dos bons hábitos de saúde bucal.

\section{Conclusão}

Apesar de a maior parte dos educadores desenvolver práticas educativas sobre saúde bucal no ambiente escolar, existe deficiência no entendimento de determinados assuntos pelos professores, fazendo-se necessária maior participação do cirurgião-dentista na orientação a esse público.

\section{Abstract}

Introduction: The oral health education is a process that aims to transform attitudes and behaviors and to form habits for the benefit of individual health. For this to occur, it is necessary to strengthen and continuous repetition, as possible in the school environment. Objective: To analyze teachers' knowledge about oral health, practices developed in the school environment, as well as the participation of dentists in guiding teachers of primary and secondary schools. Subjects and method: We used a semi-structured questionnaire with open and closed questions about teacher training, received guidance on oral health during their training, development of oral health issues in the classroom and knowledge on the subject. Results: Of the total respondents $(n=221)$, only $77(34.8 \%)$ said they had guidance on oral health in their training to be a teacher. $70(31.7 \%)$ said they did not develop oral health issues in the classroom and 91 (41.2\%) stated no interaction between dentist and professor. We observed deficiency in teachers' knowledge about certain issues related to dental health. There was statistically significant association between the dentist's guidance to teachers and the development of oral health actions and the teacher's knowledge about the location of the first permanent molar eruption $(p<0.01)$. Conclusion: This study conclude that although most educators develop educational practices on oral health in the school environment, there is a deficiency in understanding by teachers of certain subjects, making it necessary a greater participation of the dentist in the transmission of technical and scientific knowledge to them.

Keywords: Faculty. Knowledge. Health education. Oral health.

\section{Referências}

1. Albamonte LIMS, Charone S, Groisman S. Análise do conteúdo de saúde bucal nos livros didáticos de ciências da primeira série do ensino fundamental. Pesq Bras Odontoped Clin Integr 2009; 9(3):295-301.

2. Brasil. Ministério da Saúde. Brasil sorridente: a saúde bucal levada a sério. 2010 [citado 2012 Abr 26]. Disponível em URL: http://conselho.saude.gov.br/ultimas_noticias/2010/ img/apresentação_281210.pdf.

3. Garbin CAS, Rovida TAS, Garbin AJÍ, Arcieri RM, Souza NP, Moimaz SAS. Saúde bucal e educação infantil: avaliação do desgaste e do acondicionamento de escovas dentárias utilizadas por pré-escolares. Rev Odontol Unesp 2012; 41(2):81-7.

4. Vasconcelos R, Matta ML, Pordeus IA, Paiva SM. Escola: um espaço importante de informação em saúde bucal para a população infantil. PGR-Pós-Grad Rev Fac Odontol 2001; $4(3): 43-51$

5. Santos PA, Rodrigues JA, Garcia PPNS. Avaliação do conhecimento dos professores do ensino fundamental de escolas particulares sobre saúde bucal. Rev Odontol Unesp 2002; 31(2):205-14.

6. Alves UM, Volschan BCG, Haas NAT. Educação em saúde bucal: sensibilização dos pais de crianças atendidas na clínica integrada de duas universidades privadas. Pesq Bras Odontoped Clin Integr 2004; 4(1):47-51. 
7. Campos L, Bottan ER, Farias J, Silveira EG. Conhecimento e atitudes sobre saúde e higiene bucal dos professores do ensino fundamental de Itapema-SC. Rev Odontol Unesp 2008; 37(4):389-94.

8. Vellozo RCADM, Queluz DP, Mialhe FL, Pereira AC. Avaliação dos conhecimentos e práticas em saúde bucal de profissionais do ensino fundamental. Pesq Bras Odontoped Clin Integr 2008; 8(2):153-8.

9. Granville-Garcia AF, Silva JM, Guinho SF, Menezes V. Conhecimento de professores do ensino fundamental sobre saúde bucal. Rev Gaúcha Odontol 2007; 55(1):29-34.

10. Petersen PE, Esheng Z. Dental caries and oral health behaviour situation of children, mothers, and school teachers in Wuhan, People's Republic of China. Int Dent J 1998; 48(3):210-6.

11. Morano Junior M, Silva CMC, Mialhe FL, Lido YJVL. Conhecimentos acerca de saúde bucal de estudantes de um curso de magistério. Pesq Bras Odontoped Clin Integr 2007; 7(2):131-7.

12. Glasrud PH, Frazier PJ. Future elementary schoolteachers' knowledge and opinions about oral health and community programs. J Public Health Dent 1988; 48(2):74-80.

13. Goel P, Shetty V. Knowledge, attitude and practice of dental caries and periodontal disease prevention among primary schoolteachers in Udupi municipality. J Indian Soc Pedod Prev Dent 1997; 15(4):124-9.

14. Flores EMTL, Drehmer TM. Conhecimentos, percepções, comportamentos e representações de saúde e doença bucal dos adolescentes de escolas públicas de dois bairros de Porto Alegre. Ciênc Saúde Colet 2003; 8(3):743-52.

15. Tomita NE, Pernambuco RA, Lauris JRP, Lopes ES. Educação em saúde bucal para adolescentes: uso de métodos participativos. Rev Fac Odontol Bauru 2001; 9(1/2):63-9.

16. Souza GB, Sá PHRN, Junqueira SR, Frias AC. Avaliação dos procedimentos coletivos em saúde bucal: percepção de adolescentes de Embu, SP. Saúde Soc 2007; 16(3):138-48.

17. Sá LO, Vasconcelos MMVB. A importância da educação em saúde bucal nas escolas de ensino fundamental: revisão de literatura. Odontol Clín-Científic 2009; 8(4):299-303.

18. Oliveira JJB, Sousa PGB, Oliveira FB, Moura SAB. Conhecimento e práticas de professores de ensino fundamental sobre saúde bucal. Int J Dent 2010; 9(1):21-7.

19. Campos JADB, Garcia PPNS. Comparação do conhecimento sobre cárie dental e higiene bucal entre professores de escolas de ensino fundamental. Cienc Odontol Bras 2004; 7(1):58-65.

20. Lascala NT. Promoção de saúde bucal. São Paulo: Artes Médicas; 1997.

21. Santos PA, Rodrigues JA, Garcia PPNS. Conhecimento sobre prevenção de cárie e doença periodontal e comportamento de higiene bucal de professores de ensino fundamental. Cienc Odontol Bras 2003; 6(1):67-74.

22. Sanabe ME, Cavalcante LB, Coldebella CR, Abreu-e-Lima FCB. Urgências em traumatismos dentários: classificação, características e procedimentos. Rev Paul Pediatr 2009; 27(4):447-51.

\section{Endereço para correspondência:}

Ronald Jefferson Martins

NEPESCO - Núcleo de Pesquisa em Saúde Coletiva,

Departamento de Odontologia Infantil e Social, Faculdade de Odontologia de Araçatuba, Universidade Estadual Paulista, Brasil.

Rua José Bonifácio, 1193, Vila Mendonça

16015-050 Araçatuba - SP

Fone: 551836363250

E-mail: rojema@foa.unesp.br

Recebido: 03/11/2013. Aceito: 21/01/2014. 